\title{
Approach to Arbitrary Transportation of suspended particles Based on Ultrasonic composite Field
}

\author{
Zhuang Long ${ }^{1, a}$, Wu Liqun ${ }^{1 . b}$, Du Xibiao ${ }^{1, \mathrm{c}}$, Zhang Linan ${ }^{1, \mathrm{~d}}$ and Zhai Zhuang ${ }^{1, \mathrm{e}}$ \\ ${ }^{1}$ School of Mechanical Engineering, Hangzhou Dianzi University, Hangzhou310018,China \\ azhuanglongww@sina.com,,wuliqun@hdu.edu.cn, 'c410985832@qq.com, \\ d2646016812@qq.com, e137059941@qq.com
}

Keywords: inner machining; ultrasonic suspension; composite field; particle transportation.

\begin{abstract}
A particle suspension transport method was proposed based on ultrasonic composite field. Firstly, the array focusing model and accumulated force equation were established based on phased array ultrasonic cells delay, then integrated with the standing wave ultrasonic field radiation force calculation method, the particle motion control model and driving force formul under the combined ultrasound field were introduced. Through the MATLAB simulation of complex ultrasound field,the factors that affected the focusing performance and control performance were analysed.Finally,arbitrary control scheme of particle montion was put forward by compound ultrasonic driving field.
\end{abstract}

\section{Introduction}

The inner machining tehchnogy based on ultrasonictransport the machning energy or tool into the body ,and directly to machine the internal structure.in our team, we used to put forward to machine the internal structure in the non-transparent materialthat wasultrasonic standing wave technolegy ${ }^{[1,8]}$, and here are several advantage comparing tolaser techonogy, the non-transparent material can bemachined ${ }^{[2,9,10,11]}$,furthermore, the severial energy effect can be used also,but the problemwas that the machining energy or the particle can move onlybetween the standing wave node, cannot move arbitary in a sequential space, it is difficult to meet the control precision requirement.In this study, the array focusing model and accumulated force equation were established based on phased array ultrasonic cells delay, then integrated with the standing wave ultrasonic field radiation force calculation method, the particle motion control model and driving force formul under the combined ultrasound field were introduced.

\section{2.the principle of phased array and the study of focused ultrosound field}

Here we introduced ultrosonic array phasedfocusemethod ${ }^{[3]}$, derived the compound sound pressure formula.

\section{1 the principle of ultrosoinc phased array focus}

The model of ultrosonic phased array focusing model is shown in Fig.1, if the number of array elements isn,and suppose the sound pressure of $\mathrm{i}$-th element in point $\mathrm{p}$ is $P_{\mathrm{i}}$, then we can derive the gross pressure as:

$$
P=\sum_{i=1}^{n} P_{i}
$$

Due to the distance between each array elementto focus point is different, we can control the focusal signal depending on the delay time, so all the signal can reach the same focuspoint simultaneously ,similaring to the principle of concave sphericaltransducer, as the study of the concave shperical transducer, the focusdepth can be shown as:

$$
F_{e}=8.16 \lambda\left(\frac{F}{D}\right)^{2}=\frac{8.16 \lambda F^{2}}{D^{2}}, 2 F / D>1
$$

Where $\lambda$ is the wave length; $F$ is focal $; D$ is the width of the transducer.focuscan beachieved if 
the phase differenceis zero,the time differenceandphase differencecan be expressed as:

$$
\Delta \varphi=2 \pi f_{0} \times \Delta t
$$

\section{2 the expression of delay time of phased array}

Phased array focused is acheieved through the controling of the delay time of phased array ${ }^{[10,12]}$, the relationship can be obtained from the geometric relationship,it can be shown in Fig. 2

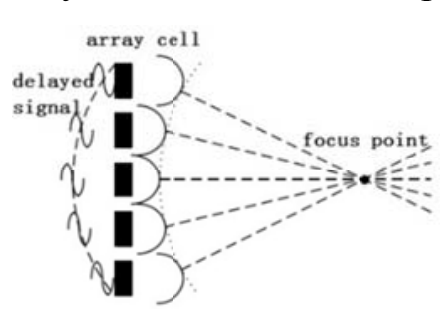

Fig.1 phased array focal model

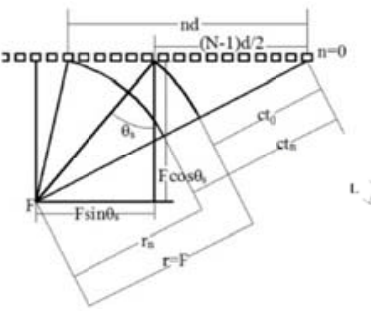

Fig. 2 phased array delay time geometry

Fig. 3 schematic of sound pressure

It derive that: $\left(F \cos \theta_{s}\right)^{2}+\left[F \sin \theta_{s}-\left(n d-\frac{N-1}{2} d\right)\right]^{2}=\left[F-\left(t_{n}-t_{0}\right) c\right]^{2}$

here, $N$ is amount of array element; $n=0,1,2,3 \ldots, N-1 ; d$ is the center space between the array cell; $D$ is the center distance of adjective array element.

then:

$$
t_{n}=\frac{F}{c}\left\{1-\left[1+\left(\frac{d}{F}\left(n-\frac{N-1}{2}\right)\right)^{2}-2 \sin \theta_{s} \frac{d}{F}\left(n-\frac{N-1}{d}\right)\right]^{1 / 2}\right\}+t_{0}
$$

the delay time between the $\mathrm{nth}$ and (n-1) cell is: $\Delta \tau_{n}=t_{n}-t_{n-1}$

It can be simplified to:

$$
\Delta \tau_{n}=\Delta \tau_{0}+\frac{c \Delta \tau_{0}^{2}}{2 F \tan ^{2} \theta_{s}}(N-2 n)
$$

here: $\Delta \tau_{0}=\frac{d \sin \theta_{s}}{c}$

\section{3 computing model of sound presssure of ultrasonic phased array focusing}

The phased array sound pressure is built as Fig.3. The distribution of sound pressure of per array can be derived as below ${ }^{[4]}$ :

$$
p=\frac{j k c u \rho_{0}}{2 \pi r} a L \frac{\sin \left(\frac{k L \sin \theta \sin \phi}{2}\right)}{\frac{k L \sin \theta \sin \phi}{2}} \frac{\sin \left(\frac{k a \sin \theta \cos \phi}{2}\right)}{\frac{k a \sin \theta \cos \phi}{2}} e^{j(\omega t-k r)}
$$

the superposition sum pressure of all the array cell is written as:

$$
p_{\mathrm{A}}=\sum_{n=0}^{n=N-1} p_{n}=\sum_{n=0}^{n=N-1} \frac{j k c u \rho_{0}}{2 \pi r} a L \frac{\sin \left(\frac{k L \sin \theta \sin \phi}{2}\right)}{\frac{k L \sin \theta \sin \phi}{2}} \frac{\sin \left(\frac{k a \sin \theta \cos \phi}{2}\right)}{\frac{k a \sin \theta \cos \phi}{2}} e^{j\left(\omega t_{n}-k r_{n}\right)}
$$

\section{4 the simulation of focus peformance of ultrasonic phased array}

In order to find the infulence of per parameter acting on the sound pressure of phased focal array ,some parameter should be determined,such as:the original demension of array element $(3 \mathrm{~mm}$ $\times 0.7 \mathrm{~mm})$, the quantity of array element $\left(N_{\mathrm{e}}=64\right)$, the spacing between array element $\left(g_{\mathrm{ap}}=0.5 \mathrm{~mm}\right)$, the initial emited frequence $(f=0.8 \mathrm{MHz})$, the focal depth was $80 \mathrm{~mm}$.the quantity of simulation array element was set to $N_{\mathrm{e}}=32,64$, and 96 ; the spacing was set to $g_{\text {ap }}=0.3 \mathrm{~mm}, 0.5 \mathrm{~mm}$, and $0.7 \mathrm{~mm}$; the frequence was set to $0.6 \mathrm{MHz}, 0.8 \mathrm{MHz}$ and $1 \mathrm{MHz}$, the sound field in different parameter is shown in Fig.4 $\left(N_{\mathrm{e}}=96\right)$,Fig. $5\left(g_{\mathrm{ap}}=0.7 \mathrm{~mm}\right)$,Fig. $6(f=1 \mathrm{MHz})$. 


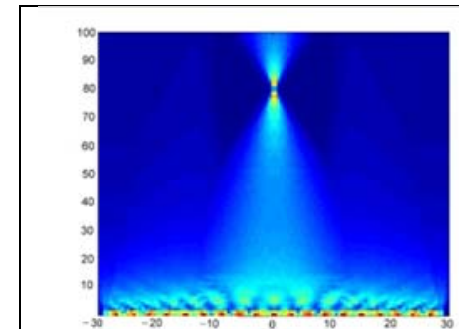

Fig. $4 N_{\mathrm{e}}=96$

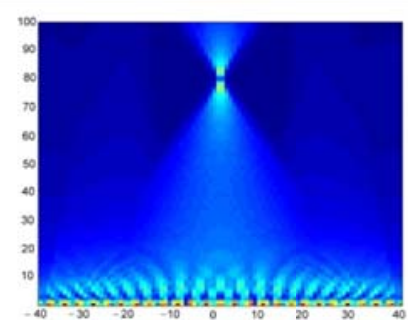

Fig. $5 g_{\text {ap }}=0.7 \mathrm{~mm}$

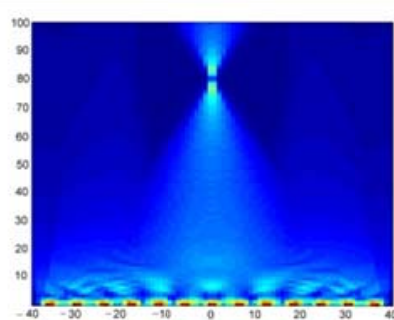

Fig. $6 f=1 \mathrm{MHz}$

the above simulation result can derive the following conclusion:Increasing the number ofarray elements,decreasing the array element spacingand the higher transmitterfrequencycan improve theperformance ofthe phasedfocus.

\section{3. driving method of ultrasonic composite field}

\section{1 ultrasonic focal radiated force}

we can assume the singal array element as a point sound source,the spherule suffered from radiation force in the sound field, the force direction went alongradial-direction, it is shouwn in Fig.7.According to thesuperposed theory ${ }^{[4]}$, the force acting on the spherule was the vector sum of each radial force ${ }^{[5,6]}$.

\subsection{1 point-p on theaxis-z}

We constructed the force relationship as Fig.8, wovassume that the centre distance of array element was $d$, the number of array element was $n$, the spherule located at point-p,the radius of spherule was $R_{\mathrm{p}}$, the plane coordinate centres o(the center of the phased array ).we analysed and calculated the forec actiong on the point-p which is respectively on theaxis- $z$ and arbitary position of plane-xoz.
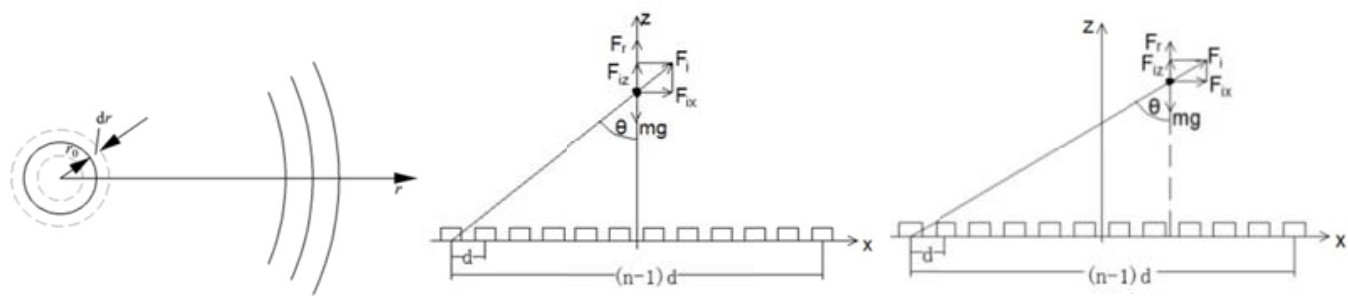

Fig.7 point sound sourceFig. 8 force onaxis-zFig.9 force onnonaxis-Z

According to the Fig.9, the ggavity and the levitation force generated by standing wave are balance in the vertical direction, the levitation force which is generated by array element, it canbedividedintotwo sub-force(axis-z and axis-x force), the resultant go along vetical direction, and the vetical forcecan be written as: $F_{i z}=\frac{b}{\sqrt{L_{i}^{2}+b^{2}}} F_{i}$

$$
\text { Here: }{ }_{F_{i}}=2 \pi \rho_{0}|A|^{2}\left(k R_{s}\right)^{6} \frac{9+\left(1-\lambda_{\rho}\right)}{9\left(2+\lambda_{\rho}\right)^{2}} ; L_{i}=\left|-\frac{(N-1)}{2} d+(i-1) d\right| ; \lambda_{\rho}=\rho_{0} / \rho_{\mathrm{p}} ;
$$

Where $b$ is coordinate value on ; $A$ is acoustic wave amplitude; $R_{\mathrm{p}}$ is the radius of particle

the driving force derived by array can be written as:

$$
F_{\mathrm{pz}}=\sum_{i=1}^{N} F_{\mathrm{iz}}=\sum_{i=1}^{N} \frac{b}{\sqrt{L_{i}^{2}+b^{2}}} F_{i}=\sum_{i=1}^{N} \frac{b}{\sqrt{L_{i}^{2}+b^{2}}} 2 \pi \rho_{0}|A|^{2}\left(k R_{\mathrm{p}}\right)^{6} \frac{9+\left(1-\lambda_{\mathrm{\rho}}\right)}{9\left(2+\lambda_{\mathrm{\rho}}\right)^{2}}
$$

\subsection{2 point-Pnon of the $z$-axis}

When the spherule is in the xozplane but ouside of the Z-axis,certainly, the force relationship haveanalysed based on the force diagram in Fig.6. the forceacted on the spherule can be divided into axis-z and axis-z force, and theaxis-z force supplied the driving force of right transportation, it can be written as: 


$$
F_{i x}=\frac{L_{i}}{\sqrt{L_{i}^{2}+b^{2}}} F_{i}
$$

here:

$$
F_{i}=2 \pi \rho_{0}|A|^{2}\left(k R_{\mathrm{p}}\right)^{6} \frac{9+\left(1-\lambda_{\mathrm{p}}\right)}{9\left(2+\lambda_{\mathrm{p}}\right)^{2}}, \quad L_{i}=a+\frac{(N-1)}{2} d-(i-1) d 。
$$

The right driving force can be written similarly as:

$$
F_{\mathrm{px}}=\sum_{i=1}^{M} F_{\mathrm{ix}}=\sum_{i=1}^{M} \frac{L_{i}}{\sqrt{L_{i}^{2}+b^{2}}} F_{i}=\sum_{i=1}^{M} \frac{L_{i}}{\sqrt{L_{i}^{2}+b^{2}}} 2 \pi \rho_{0}|A|^{2}\left(k R_{\mathrm{p}}\right)^{6} \frac{9+\left(1-\lambda_{\mathrm{p}}\right)}{9\left(2+\lambda_{\mathrm{p}}\right)^{2}}(4)
$$

where $M$ is the quantity of array element;we defined that only the left array element work;we can derive that:

$$
a+\frac{(N-1)}{2} d-(i-1) d \geq 0
$$

Then we can get: $i \leq \frac{a}{d}+\frac{N+1}{2}$, where $M=\left\lfloor\frac{a}{d}+\frac{N+1}{2}\right\rfloor$, that is the upper limit.where, \lfloor\rfloor is shown the maximum integer which does not exceed the number.

Similarly, we can derive the axis-z driving force as below:

$$
F_{\mathrm{pz}}=\sum_{i=1}^{M} F_{i z}=\sum_{i=1}^{M} \frac{b}{\sqrt{L_{i}^{2}+b^{2}}} F_{i}=\sum_{i=1}^{M} \frac{b}{\sqrt{L_{i}^{2}+b^{2}}} 2 \pi \rho_{0}|A|^{2}\left(k R_{\mathrm{s}}\right)^{6} \frac{9+\left(1-\lambda_{\mathrm{\rho}}\right)}{9\left(2+\lambda_{\mathrm{\rho}}\right)^{2}}
$$

If the spherule suffered the right side driving force that generated by right side array element,similarly,we can get the left side drving force.

\section{2 the caculation of standing waveradiation force of particle}

Combinding the expression of the sound pressrue with the condition of boundary,we can obtain the expression of radiation force.KING ${ }^{[6]}$,Yosioka and Kawasima ${ }^{[7]}$ have demonstrated the theory of sound radiation force, and the expression can be written as:

$$
\begin{array}{r}
F_{\mathrm{r}}=-\left(\frac{\pi p_{0}^{2} V_{\mathrm{p}} \beta_{\mathrm{m}}}{2 \lambda}\right) \phi(\beta, \rho) \sin (2 k x) ; \\
\phi(\beta, \rho)=\frac{5 \rho_{p}-2 \rho_{m}}{2 \rho_{p}+\rho_{m}}-\frac{\beta_{p}}{\beta_{m}}
\end{array}
$$

Where, $V_{\mathrm{p}}$ is the volume of spherule, $\rho_{\mathrm{p}}$ is density of spherule, $\rho_{\mathrm{m}}$ is thedensity of medium, $\beta_{\mathrm{p}}$ is the compressible coefficient of spherule, $\beta_{\mathrm{m}}$ is the compressible coefficient of medium, and the compressible coefficient is related with the speed of wave velocity of medium, it can be written as:

$$
=\beta 1 / \rho c^{2}
$$

\section{3 physical model of ultrasonic composite field}

the array element of high-frequency ultrasonic standing wav was embeded on the endsurface of ultrasonic amplitude amplifer of low-frequency(in the Fig.10), this setup formed a ultrasonic composite field(UCF), and the radiation force on which acted on the spacial particle in the UCF is the vector sum of standing wave and phased array element,that is:

$$
F_{\mathrm{c}}=F_{\mathrm{r}}+F_{\mathrm{p}}(7)
$$



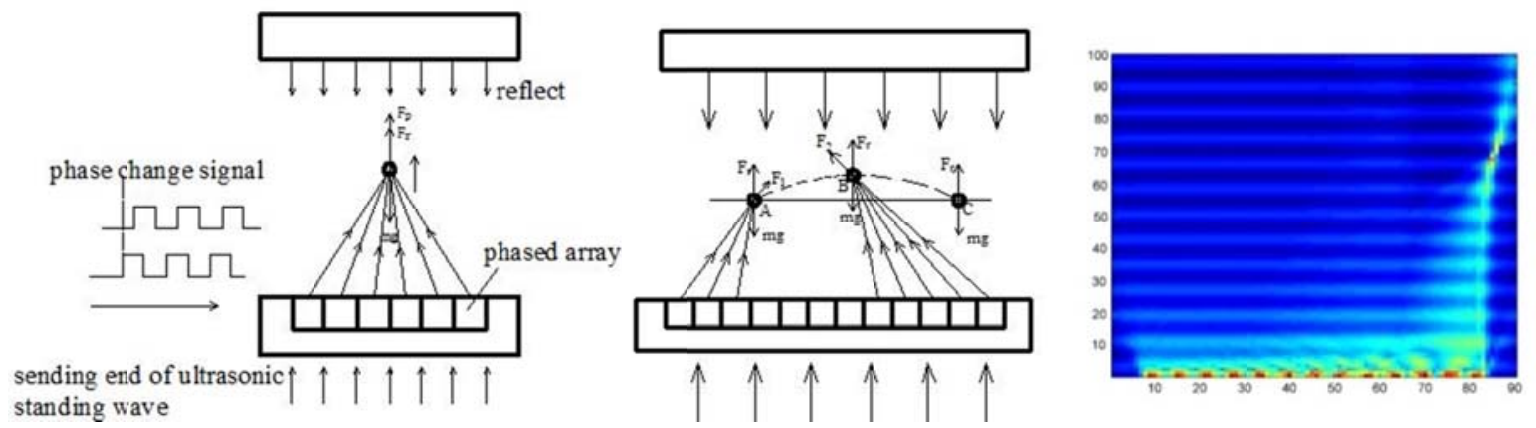

Fig.10 vertical motionFig.11 horizontal motionFig.12 horizontal direction driving

\section{4. study of particle transportion in the UCF}

\section{1 particle transportion in veticle direction}

We can adjust the phase and frequence of the standing wave to control the particle motion.we can adjust the parameter of the standin wave and the phased array as well.

\section{2 particle transportion in horizontal direction}

the array element located in the opposite direction of particle motion are triggered,according to the above study(in he Fig.10)the particle suffered the force from the vetical and horizontal direction.the spherule will keep dynamic stabele in the vertical direction due to the restroring force will balance the standing wave force.then thehorizontal force is the main driving force,the superposed horizontal force generated by parts of the array element will drive the spherule motin, it is shown in the belowig.11.we $\mathrm{c}$ an adjust the delay time to control the particle motion in the UCF, it is shown in the Fig.12, the simlation demonstratet that the controlable sound pressure can be achieve near the focal point ,and this can adjust dynamicly the force situation actiong on the spherule.

\section{5. conclusion}

This paper introduced the UCF theory,the simulation result proved that the implementation of a wide range of space suspension transportation is workable.the arbitary focus characteristic of phased array will balance the restoring force, establish dynamic balance expression and achieve levitation driving of particle , the driving method can achieve arbitary suspension transportion ,and the shift of displacement was obtained by adjusting the deray time, the UCF technology will simlify the controlling algorithm, improve the controlling accuracy of transportion,provide greater flexibility and handling for the inner machning technology, the next work we will construct the experiment system and anasys the phased delay arithmetic ${ }^{[13]}$.

\section{Acknowledgements}

This wrok was financially supported by the National Natural Science Foundation of China ( 51175134 andZhejiang Provincial Natural Science Foundation of China（LZ15E050004;

\section{References}

[1]Foresti, D., Nabavi, M., Klingauf, M., Ferrari, A. \& Poulikakos, D.

Acoustophoretic contactless transport and handling of matter in air. Proc. Natl Acad. Sci. USA 110, 12549-12554 (2013).

[2] Mu. F, Zhang .Y.Technical research of 3D micro-channel system, Journal of China Academy of Electronics and Information Technology.(2011)20-23.

[3]Li.J. research on Acoustic focus phased array.Shanxi normal university,2003.

[4]Du.G,Zhu.Z,Gong.X.Fundamentals of coustic.in,Nanjing university,2001.

[5]GOR'KOV L P. On the forces acting on a small particle in an acoustical field in an ideal fluid [J]. 
Sov. Phys. Dokl, 1962, 6: 773-775.

[6]KING L V. On the acoustic radiation pressure on spheres.Proc. R. Soc. London, Ser. A, Math. Phys. Sci, 1934, 147(861): 212-240.

[7]YOSIOKA K, and KAWASIMA Y. Acoustic radiation pressure on a compressible sphere. Acustica, 1955, 5: 167-173.

[8]Whymark, R. R. Acoustic field positioning for containerless processing. Ultrasonics 13, 251-261 (1975).

[9]VANDAELE V, DELCHAMBRE A, LAMBERT P. Acoustic wave levitation: Handling of components. J. Appl. Phys, 2011, 109(12): 124901-124901-7.

[10]Fan.Z. Research on key technologies of noncontact manipulation for micro-components based on ultrasonic radiation force field, in, Zhejiang University, 2011.

[11]Takayuki H,Yoichi O,Jun R.Three-dimensional noncontact manipulation by opposite ultrasonic phased arrays.Japanese Journal of Applied Physics, 2014 , 07 KE07.

[12]Foresti, D. \& Poulikakos, D. Acoustophoretic contactless elevation, orbital transport and spinning of matter in air. Phys. Rev. Lett. 112, 024301 (2014).

[13]Ochiai, Y, Hoshi, T. \& Rekimoto, J. Pixie dust: graphics generated by levitated and animated objects in computational acoustic-potential field. ACM Trans.Graph. 33, 85 (2014). 